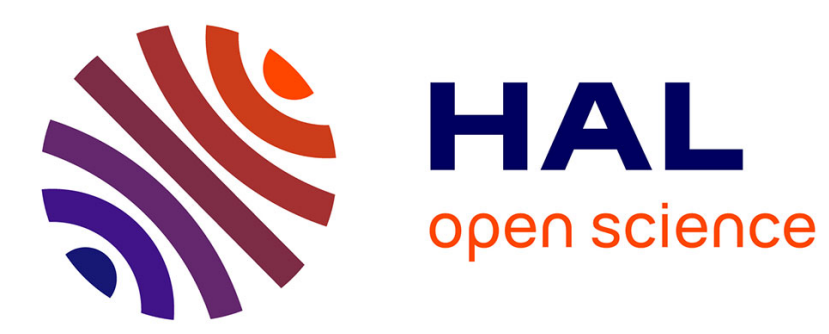

\title{
On the Simplifications of Cable Model in Static Analysis of Large-Dimension Cable-Driven Parallel Robots
}

Dinh Quan Nguyen, Marc Gouttefarde, Olivier Company, François Pierrot

\section{To cite this version:}

Dinh Quan Nguyen, Marc Gouttefarde, Olivier Company, François Pierrot. On the Simplifications of Cable Model in Static Analysis of Large-Dimension Cable-Driven Parallel Robots. IROS: Intelligent RObots and Systems, Nov 2013, Tokyo, Japan. pp.928-934, 10.1109/IROS.2013.6696461 . lirmm01221398

\author{
HAL Id: lirmm-01221398 \\ https://hal-lirmm.ccsd.cnrs.fr/lirmm-01221398
}

Submitted on 28 Oct 2015

HAL is a multi-disciplinary open access archive for the deposit and dissemination of scientific research documents, whether they are published or not. The documents may come from teaching and research institutions in France or abroad, or from public or private research centers.
L'archive ouverte pluridisciplinaire HAL, est destinée au dépôt et à la diffusion de documents scientifiques de niveau recherche, publiés ou non, émanant des établissements d'enseignement et de recherche français ou étrangers, des laboratoires publics ou privés. 


\title{
On the Simplifications of Cable Model in Static Analysis of Large-Dimension Cable-Driven Parallel Robots
}

\author{
Dinh Quan Nguyen, Marc Gouttefarde, Olivier Company and François Pierrot*
}

\begin{abstract}
This paper addresses the simplification of cable model in static analysis of large-dimension cable-driven parallel robots (CDPR). An approach to derive a simplified hefty cable model is presented. The approach provides an insight into the limitation of such a simplification. The resulting cable tension computation is then used to solve the inverse kinematic problem of CDPR. A new expression of cable length taking into account both the non-negligible cable mass and elasticity is also introduced. Finally, simulations and experiments on a large CDPR prototype are provided. The results show that taking into account both cable mass and elasticity improves the robot accuracy.
\end{abstract}

\section{INTRODUCTION}

In the past twenty years, cable-driven parallel robots (CDPR) have been extensively studied in favor of their appealing advantages such as light-weight, simple in design and relatively low cost. Compared to parallel manipulators with rigid links, CDPR use cables to position their endeffector directly by controlling the length of each cable. Possible applications in manufacturing industry, construction industry and aerospace industry are positioning and handling of large and heavy parts across wide workspaces. Several studies on large-dimension CDPR have been made [1]-[15].

In many previous studies on CDPR, all cables have been considered massless. This assumption is usually valid for robots of reasonable size and carrying light payloads, but for large-dimension robots or for robots that carry heavy payloads, such an assumption may be invalid [13]. Irvine presents in [16] the well-known cable model known as the elastic catenary which takes the cable mass and elasticity into account. In [17], a cable catenary model in 3D Cartesian space is introduced. Because of their highly nonlinear nature, numerical methods must be considered to deal with the catenary cable modeling. However, these methods are usually relatively slow because of the involved computations. Therefore, using such a cable model for CDPR may be impractical in real-time environments, especially in solving the inverse kinematics and tension distribution problems. Finding means of reducing the complexity of such cable models is thus of great practical interest.

Over the past decades, the methods to deal with the catenary cable modeling have been simplified and improved. In [16], a parabolic cable model is presented. It is valid

\footnotetext{
* Dinh Quan Nguyen, Marc Gouttefarde, Olivier Company and François Pierrot are with the Laboratoire d'Informatique, de Robotique et de Microélectronique de Montpellier (LIRMM-CNRS-UM2), 161 rue Ada, 34392 Montpellier Cedex 5, France dinhquan.nguyendirmm.fr, marc.gouttefardedirmm.fr, companydirmm.fr, pierrotelirmm.fr
}

if the sagging of the cable is small enough. However, the suggested validity condition is not explicitly derived. In [7], Kozak presents a method to solve the inverse kinematics of general CDPR when the cable model is the elastic catenary. An expression for the unstrained cable length, which takes into account the cable mass but without considering the cable elasticity, is provided. Rui Yao et al. introduce in [12] the large-dimension four-cable-driven parallel robot in FAST in which the parabolic cable model known in [16] is used to obtain an expression for the cable length. However, the latter is just an approximation of the one presented in [7] and does not account for the cable elasticity. In [13], a simplified static analysis of large-dimension CDPR is introduced based on the parabolic hefty cable model presented in [16]. The determination of the cable tension distribution is thereby transformed into one that is similar in form to the case of CDPR with massless cables. The domain of validity of the analysis proposed in [13] was left as an open issue. Later, in [14], Hui Li et al. give a relationship between the cable horizontal and vertical force components which neglects the elasticity effect from the catenary cable model. No additional development of the cable model from this relationship are proposed by these authors.

Efficient means to find practical forms of cable model have been proposed. Nevertheless unsolved issues still exist. Some of them are the validity of the simplified static analysis proposed in [13] and the precision of the inverse kinematic solutions given in [7]. The motivation of the present paper comes directly from these issues. First of all, a rigorous simplification procedure of cable catenary model in static analysis is presented. It provides a linear relationship between the cable force components and gives a clear view of the involved simplifications as well as the limitation of the method. Then, a solution for the computation of cable length is demonstrated. To the best of our knowledge, the resulting expression of the cable length is new. It takes into account both the non-negligible mass and elasticity of the cable.

The paper is organized as follows. The well-known cable catenary is recalled in Section II. The rigorous simplification steps are introduced in Section III and leads to the same parabolic simplified cable model as the one presented in [16]. This model leads directly to a linear relationship between cable horizontal and vertical force components. The limitation of the simplified cable model is discussed in Section IV. The new expression of the cable length taking into account both the cable mass and elasticity is derived in Section V. Finally, Section VI presents simulation and experimental results obtained for a large-dimension 6-DOF 


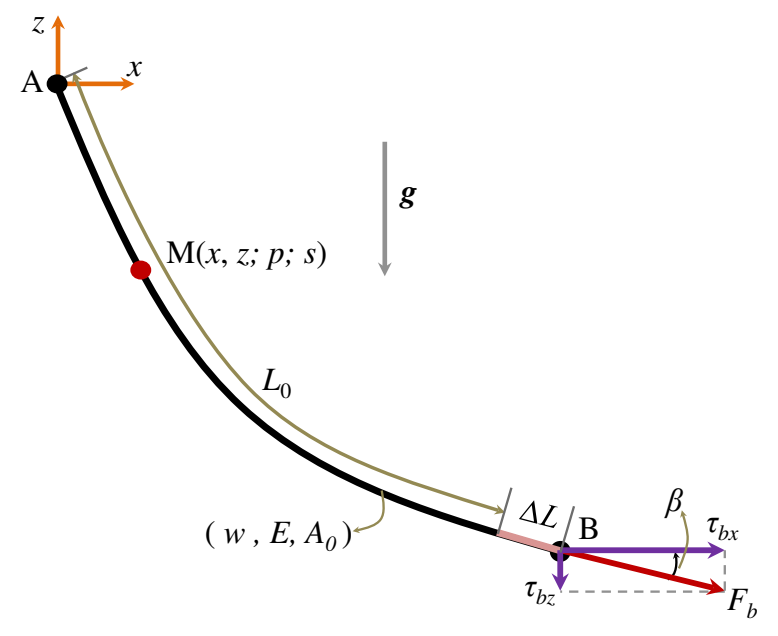

Fig. 1: Diagram of a sagging cable

parallel robot driven by $m=8$ cables.

\section{CABLE PROFILE}

Let us consider a steel cable that has unstrained length $L_{0}(m)$, self-weight $w(N / m)$, elastic modulus $E(P a)$ and cable cross-section area $A_{0}\left(\mathrm{~m}^{2}\right)$.

Fig. 1 shows the relevant coordinates and parameters of a cable lying in a vertical plane in static equilibrium. The cable is fixed between two end-points $A\left(x_{a}, z_{a}\right)$ and $B\left(x_{b}, z_{b}\right)$. All the coordinates are in the local frame attached to the vertical plane containing the cable. The term $\Delta L$ represents the strain of the cable.

A point $M$ along the strained cable has Cartesian coordinates $x$ and $z$. The variable $p$ represents the strained length of the cable segment as measured from the end-point $A$ of the cable to the point $M$. The variable $s$ will be used to denote the unstrained length of the same cable segment. In Fig.1, the origin of the local frame will be placed at the end-point $A$. Thus, the variable $s$ lies in the range:

$$
0 \leq s \leq L_{0}
$$

with $M(s=0) \equiv A$ and $M\left(s=L_{0}\right) \equiv B$.

The well-known catenary equations can be written as follows [16]:

$$
\begin{aligned}
x(s)=x_{b} & +\frac{\tau_{b x}\left(s-L_{0}\right)}{E A_{0}} \\
& +\frac{\tau_{b x}}{w} \ln \left[\frac{\tau_{s}+\tau_{b z}+w\left(s-L_{0}\right)}{\tau_{b}+\tau_{b z}}\right] \\
z(s)=z_{b} & +\frac{\tau_{b z}\left(s-L_{0}\right)}{E A_{0}} \\
& +\frac{w\left(s-L_{0}\right)^{2}}{2 E A_{0}}+\frac{1}{w}\left(\tau_{s}-\tau_{b}\right)
\end{aligned}
$$

where $\tau_{b x}, \tau_{b z}$ are the cable horizontal and vertical force components at point $B$ and $\tau_{s}, \tau_{b}$ are the tensions in cable at point $M$ and $B$, respectively:

$$
\tau_{s}=\sqrt{\tau_{b x}^{2}+\left[\tau_{b z}+w\left(s-L_{0}\right)\right]^{2}}
$$

$$
\tau_{b}=\sqrt{\tau_{b x}^{2}+\tau_{b z}^{2}}
$$

The shape of the cable must satisfy the geometric constraint:

$$
\left(\frac{d x}{d p}\right)^{2}+\left(\frac{d z}{d p}\right)^{2}=1
$$

which implies:

$$
\frac{d p}{d x}=\sqrt{1+\left(\frac{d z}{d x}\right)^{2}}
$$

At point $M$, the force balance for the segment of the cable between points $M$ and $B$ can be written as follows:

$$
\begin{aligned}
\tau_{s}\left(\frac{d x}{d p}\right) & =\tau_{b x} \\
\tau_{s}\left(\frac{d z}{d p}\right) & =\tau_{b z}+w\left(s-L_{0}\right)
\end{aligned}
$$

The cable tension at point $M$ is considered to satisfy Hooke's law:

$$
\tau_{s}=E A_{0}\left(\frac{d p}{d s}-1\right)
$$

The relationship between coordinates $x$ and $z$ which does not depend explicitly on the variable $s$ can be derived from (5), (6) and (7):

$$
\frac{d^{2} z}{d x^{2}}=\frac{w}{\tau_{b x}} \cdot \frac{E A_{0}}{E A_{0}+\tau_{s}} \cdot \sqrt{1+\left(\frac{d z}{d x}\right)^{2}}
$$

Let us assume that $\tau_{s} \ll E A_{0}$. Then, the cable elasticity has a very little influence on the cable shape since (8) can be reduced to the simpler expression:

$$
\frac{d^{2} z}{d x^{2}}=\frac{w}{\tau_{b x}} \cdot \sqrt{1+\left(\frac{d z}{d x}\right)^{2}}
$$

The solution of (9) is:

$$
z(x)=\frac{\tau_{b x}}{w} \cosh \left(\frac{w}{\tau_{b x}} x+C_{1}\right)+C_{2}
$$

which must satisfy the following boundary conditions:

$$
\begin{aligned}
& z\left(x_{a}\right)=z_{a} \\
& z\left(x_{b}\right)=z_{b}
\end{aligned}
$$

As the origin of the local cable frame has been chosen at the end-point $A, x_{a}=0$ and $z_{a}=0$, then:

$$
\begin{gathered}
C_{2}=-\frac{1}{\mu} \cosh \left(C_{1}\right) \\
z_{b}=\frac{1}{\mu}\left[\cosh \left(\mu x_{b}+C_{1}\right)-\cosh \left(C_{1}\right)\right]
\end{gathered}
$$

where

$$
\mu=\frac{w}{\tau_{b x}}
$$

The term $C_{1}$ can be found by solving (13):

$$
C_{1}=\ln \left(\frac{\sqrt{\mu^{2} z_{b}^{2}+e^{\mu x_{b}}+e^{-\mu x_{b}}-2}+\mu z_{b}}{e^{\mu x_{b}}-1}\right)
$$


The condition to achieve (15) are:

$$
\begin{aligned}
& x_{b}>0 \\
& \tau_{b x}>0
\end{aligned}
$$

(16a) is verified by definition of the local cable frame. (16b) is always true because the cable can only pull on the mobile platform. Note that (15) is the only solution of (13) because conditions (16a) and (16b) are always verified. The tangent at point $\mathrm{B}$ of the cable is then computed as:

$$
\tan (\beta)=\left.\frac{d z}{d x}\right|_{x=x_{b}}=\frac{\tau_{b z}}{\tau_{b x}}=\sinh \left(\mu x_{b}+C_{1}\right)
$$

Relationship (17) is similar to the one presented in [14]. Note that the simplifying assumption $\left(E A_{0} \gg \tau_{s}\right)$ and the validity conditions (16a) and (16b) are not explicited in [14].

\section{LINEARIZATION}

In the case of cable-driven parallel robots with massless cables, several methods have been developed to solve important issues such as the tension distribution problem for 6-DOF CDPR with a number of cables $m \geq 6$, e.g. in [19] and [20]. To take advantage of such methods, as presented in [13], in case of CDPR with cables of non-negligible mass, one must achieve a linear relationship between the horizontal and vertical cable force components in order to transform the equilibrium equations of the mobile platform into ones similar to the case of massless CDPR. From (17), since $\tan (\beta)$ is a function of the cable horizontal force component $\tau_{b x}$, the relationship between the cable force components at end-point $\mathrm{B}$ is a nonlinear relationship $\left(\tau_{b z}=\tan (\beta) \cdot \tau_{b x}\right)$. Hence, simplification of the term $\tan (\beta)$ needs to be performed in a proper way. To this end, one can consider the Taylor series expansion of (17) in term of the variable $\mu$ defined in (14).

In fact, choosing $\mu$ as the expansion variable is reasonable since, in the case of a CDPR with steel cables carrying heavy payloads, $\mu$ is relatively small and depends only on the cable self-weight $w$ and the cable horizontal force component $\tau_{b x}$. The Taylor series expansion of $\tan (\beta)$ around the expansion point $\mu=0$ up to order of 3 is:

$$
\begin{aligned}
\tan (\beta) & =-\sinh (H)+\frac{1}{2} x_{b} \cosh (H) \mu \\
& -\left[\frac{x_{b}^{2} z_{b} \cosh (H)}{24 \sqrt{x_{b}^{2}+z_{b}^{2}}}+\frac{1}{8} x_{b}^{2} \sinh (H)\right] \mu^{2} \\
& +\left[\frac{1}{48} x_{b}^{3} \cosh (H)+\frac{x_{b}^{3} z_{b} \sinh (H)}{48 \sqrt{x_{b}^{2}+z_{b}^{2}}}\right] \mu^{3}+O\left(\mu^{4}\right)
\end{aligned}
$$

where

$$
H=\ln \left(\frac{x_{b}}{\sqrt{x_{b}^{2}+z_{b}^{2}}+z_{b}}\right)
$$

In order to achieve a linear relationship between the cable force components $\tau_{b x}$ and $\tau_{b z}$, only expansion of order 1 in (18) is to be considered:

$$
\begin{aligned}
\tan (\beta) & =-\sinh (H)+\frac{1}{2} x_{b} \cosh (H) \mu \\
& =\frac{z_{b}}{x_{b}}+\frac{1}{2} \sqrt{x_{b}^{2}+z_{b}^{2}} \mu
\end{aligned}
$$

Finally, we achieve the linear relationship:

$$
\tau_{b z}=\tan \left(\beta_{0}\right) \cdot \tau_{b x}+\frac{w L}{2}
$$

where $\tan \left(\beta_{0}\right)=z_{b} / x_{b}$ and $L=\|A B\|=\sqrt{x_{b}^{2}+z_{b}^{2}}$.

Relationship (20) is the same as the simplified cable model presented in [13].

In summary, we have rigorously detailed the simplifications to the elastic catenary cable modeling which lead to a linear relationship between the horizontal $\tau_{b x}$ and vertical $\tau_{b z}$ cable force components in (20). This linear relationship is the basis of the simplified static analysis introduced in [13]. The first assumption $\tau_{s} \ll E A_{0}$ was made in (8). If verified, it means that the cable elasticity has no real influence on the cable shape. The second assumption states that the variable $\mu$ defined in (14) is small enough. Depending on the context, the validity of these two assumptions may be an issue so that they should be carefully checked.

\section{LIMITATION OF THE SIMPLIFIED CABLE MODEL}

In the linearization procedure presented in Section III, if the expansion variable is chosen as $\nu=\mu x_{b}$ instead of $\mu$, and the Taylor series expansion of $\tan (\beta)$ is performed around the expansion point $\nu=0$, we obtain the same linear relationship (20). Hence, the accuracy of the approximation of $\tan (\beta)$ should also be justified in term of the variable $\nu$ since $\nu>\mu$ for large-dimension CDPR ( $x_{b}$ can be large). Equation (20) is valid if $\nu$ is small enough:

$$
0 \leq \nu \leq \nu_{\min }
$$

where $\nu_{\min }$ is a chosen small value. This condition leads to the following constraint on the cable horizontal force component:

$$
\frac{w x_{b}}{\nu_{\min }} \leq \tau_{b x}
$$

The validation of condition (22) gives an insight into the limitation of the simplified cable model. To illustrate this point, let us consider the problem which consists in determining the wrench-feasible workspace (WFW) [18] of a CDPR driven by $m$ cables. The WFW is the set of poses of the mobile platform for which, for any wrench $\mathbf{f}$ in $W_{\text {req }}$, there exists a vector of cable horizontal force components $\tau_{\mathbf{b x}}$ which is a solution to the equilibrium equations

$$
\mathbf{W} \tau_{\mathbf{b x}}=\mathbf{f}
$$

and verifies

$$
\tau_{\min } \leq \tau_{b x i} \leq \tau_{\max }(i=\overline{1, m})
$$

Here, $W_{\text {req }}$ is the required set of wrenches that the cables must apply at the reference point $P_{c}$ of the mobile platform. Equation (23) is the modified equilibrium equations of the mobile platform obtained with the simplified static analysis presented in [13] which assumes that (20) is valid. For a given CDPR geometry, the wrench matrix $\mathbf{W}$ depends only on the pose of the mobile platform. If the required set of 
wrenches $W_{\text {req }}$ is also known, then the size of the $W F W$ is determined by condition (24).

For the simplified static analysis of [13] to be valid accross the $W F W$, the lower bound of (24) must not be smaller than

$$
\tau_{\min }=\frac{w x_{b}}{\nu_{\min }}
$$

The size of the $W F W$ is proportional to the admissible range $\left[\tau_{\min }, \tau_{\max }\right]$ of the cable horizontal force component. In fact, the upper bound on the cable forces is always determined by safety considerations. Hence, with a fixed upper bound on the cable horizontal force component $\left(\tau_{\max }=\right.$ const $)$, the size of the part of the $W F W$ in which the simplified cable model is valid is reduced when $\tau_{\min }$ increases. That makes the size of this workspace inversely proportional to $x_{b}$ (and also to the cable self-weight $w$ ).

In practice, it is preferable to have a large $W F W$. For a given set of wrenches and in a large desired $W F W$, condition (22) may be violated in some areas. Thus, it is expected to get large errors in the approximation of the term $\tan (\beta)$. Such errors lead to a poor accuracy in solving the cable tension distribution problem with the simplified static analysis of [13]. The problem is even more involved since the assumption $\tau_{s} \ll E A_{0}$ may be questioned if $\tau_{b x}$ is large.

\section{COMPUTATION OF THE CABLE LENGTH}

Starting from (7), one can derive:

$$
\begin{aligned}
d p & =\left(1+\frac{\tau_{s}}{E A_{0}}\right) d s=\left(1+\frac{\tau_{b x}}{E A_{0}} \cdot \frac{d p}{d x}\right) d s \\
\frac{d p}{d x} & =\left(1+\frac{\tau_{b x}}{E A_{0}} \cdot \frac{d p}{d x}\right) \frac{d s}{d x}
\end{aligned}
$$

Substitute $d p / d x$ from (5) into (26) to obtain:

$$
\frac{d s}{d x}=\frac{\sqrt{1+\left(\frac{d z}{d x}\right)^{2}}}{1+\frac{\tau_{b x}}{E A_{0}} \sqrt{1+\left(\frac{d z}{d x}\right)^{2}}}
$$

If we use the solution of $z(x)$ from (10) and since,

$$
\sqrt{1+\left(\frac{d z}{d x}\right)^{2}}=\cosh \left(\mu x+C_{1}\right)
$$

where $C_{1}$ is given in (15), then (27) becomes:

$$
\frac{d s}{d x}=\frac{E A_{0}}{\tau_{b x}}\left(1-\frac{1}{1+R}\right)
$$

where

$$
R=\frac{\tau_{b x}}{E A_{0}} \cosh \left(\mu x+C_{1}\right)
$$

Since $R \ll 1$ because we assume that $\tau_{b x} \ll E A_{0}$, let us use the Taylor series expansion to find an approximation of $d s / d x$ around the expansion point $R=0$ as:

$$
\frac{d s}{d x}=\frac{E A_{0}}{\tau_{b x}}\left[R-R^{2}+R^{3}+O\left(R^{4}\right)\right]
$$

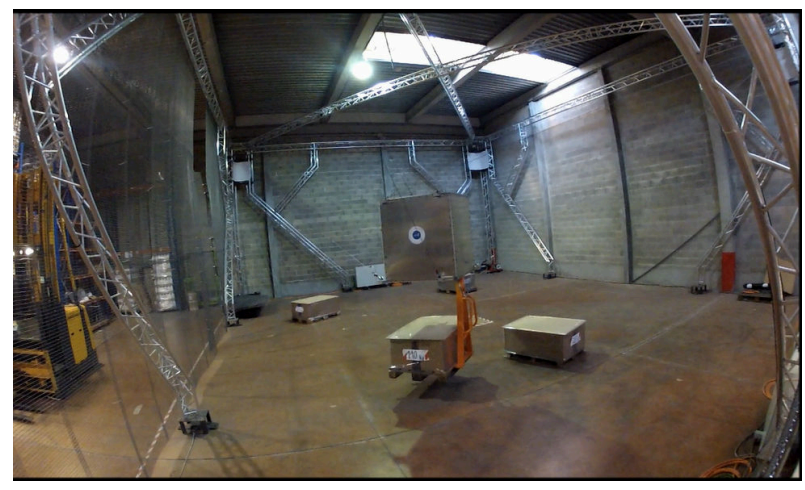

Fig. 2: LIRMM/Tecnalia CoGiRo prototype of global dimensions $15 m \times 11 m \times 6 m(l \times w \times h)$

The cable unstrained length can finally be obtained as follows:

$$
\begin{array}{r}
L_{0}=\int_{0}^{L_{0}} d s=\int_{x_{a}=0}^{x_{b}} \frac{E A_{0}}{\tau_{b x}}\left[R-R^{2}+R^{3}\right] d x \\
=\frac{1}{\mu}\left[\sinh \left(C_{b}\right)-\sinh \left(C_{1}\right)\right] \\
-\frac{\tau_{b x}}{4 E A_{0} \mu}\left[2 \mu x_{b}-\sinh \left(2 C_{1}\right)+\sinh \left(2 C_{b}\right)\right] \\
-\frac{\tau_{b x}^{2}}{12 E^{2} A_{0}^{2} \mu}\left[9 \sinh \left(C_{1}\right)-9 \sinh \left(C_{b}\right)\right. \\
\left.+\sinh \left(3 C_{1}\right)-\sinh \left(3 C_{b}\right)\right]
\end{array}
$$

with $C_{b}=\mu x_{b}+C_{1}$.

Let us note that, in (31), if the series expansion of $d s / d x$ only up to order 1 of $\mathrm{R}$ is considered, the expression of $L_{0}$ is the same as the one presented in [7] where only the cable mass is taken into account (elasticity neglected).

The unstrained cable length as given in (31) is the one needed for the inverse kinematics and thus for the trajectory planning part in a control scheme of a CDPR.

\section{VALIDATION}

The validation of the simplified cable model with the new cable length expression (31) is performed in simulation and experimentally on the CoGiRo prototype, a large-dimension suspended CDPR driven by $m=8$ cables shown in Fig. 2. The characteristics of the steel cables driving the mobile platform are:

$$
\begin{array}{ll}
\circ & E=35 \mathrm{e} 9(\mathrm{~Pa}) \\
\circ & A_{0}=8.2051 \mathrm{e}-06\left(\mathrm{~m}^{2}\right) \\
\circ & w=0.62784(\mathrm{~N} / \mathrm{m})
\end{array}
$$

\section{A. Simulation}

The center of mass $P_{c}$ of the mobile platform of the robot follows a desired trajectory in the Cartesian space $X_{t}=\left(\begin{array}{lll}x_{t} & y_{t} & z_{t}\end{array}\right)$ with constant orientation $\alpha_{x, y, z}=\left(\begin{array}{lll}0 & 0 & 0\end{array}\right)$ (X-Y-Z Euler angle convention). The mass of the mobile platform is $m_{p}=500 \mathrm{~kg} . X_{t}$ is the position of $P_{c}$ at time $t$. Smoothed trapezoidal velocity method [22] is used to generate the desired trajectory. The robot is simulated 

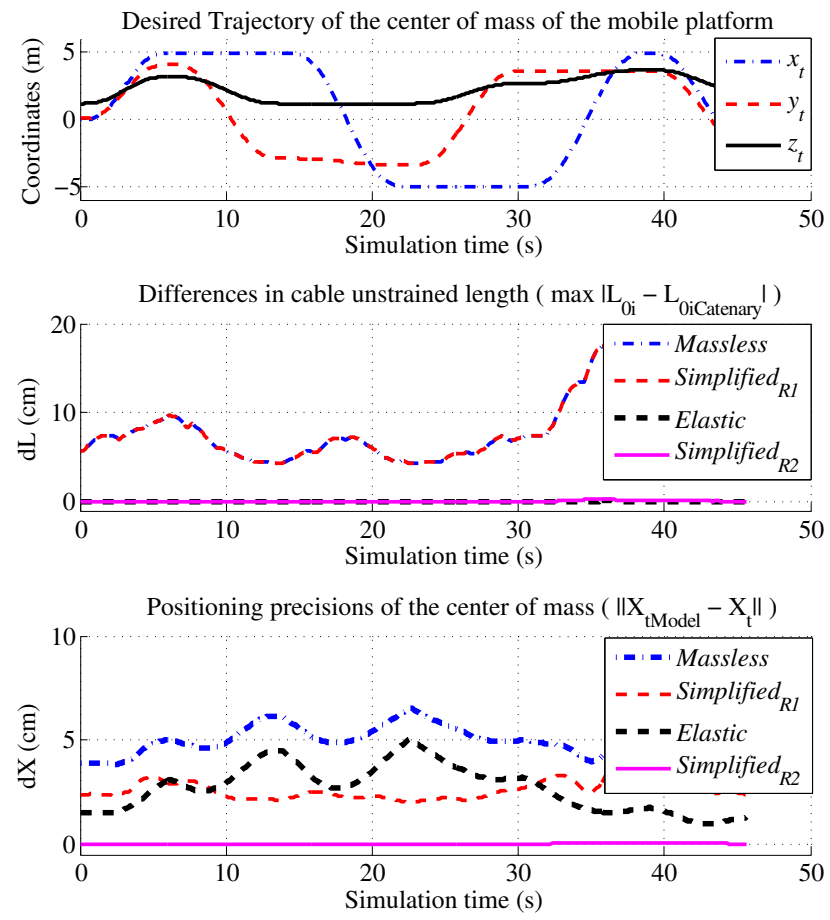

Fig. 3: Accuracies of different cable models

Case 1a : following a desired trajectory

using the massless cable model $\left(L_{0 i M a s s l e s s}=\left\|A_{i} B_{i}\right\|\right)$, the elastic cable model (where only elasticity is considered) and the simplified hefty cable model with different expressions of cable unstrained length corresponding to Taylor series expansion of $L_{0}$ up to order 1 and 2 of $\mathrm{R}$ in (31). The cable unstrained lengths of the elastic cable model are computed as follows:

$$
L_{0 i \text { Elastic }}=\frac{L_{0 i M a s s l e s s}}{1+\frac{\tau_{i}}{E A_{0}}} \quad(i=\overline{1, m})
$$

where $\tau_{i}$ is the cable tension of the $i$-th cable obtained in the case of massless cable model.

Different criteria are used to evaluate the results:

$$
\begin{gathered}
d L=\max _{1 \leq i \leq m}\left|L_{0 i}-L_{0 i \text { Catenary }}\right| \\
d X_{\text {Model }}=\left\|X_{\text {tModel }}-X_{t}\right\|
\end{gathered}
$$

where $L_{0 i}$ is the unstrained length of the $i$-th cable in the cases of the massless, elastic and simplified hefty cable models. $L_{0 i \text { Catenary }}$ is the unstrained length of the $i$-th cable in case of the elastic catenary cable model. $X_{t M o d e l}$ is the simulated position of $P_{c}$ at time $t$ obtained for the CDPR with a given cable model.

The variable $\nu$ (introduced in Section IV) is computed in case of the simplified hefty cable models to illustrate the discussion of Section IV:

$$
\nu_{\max }=\max _{1 \leq i \leq m} \frac{w x_{b i}}{\tau_{b x i}}
$$
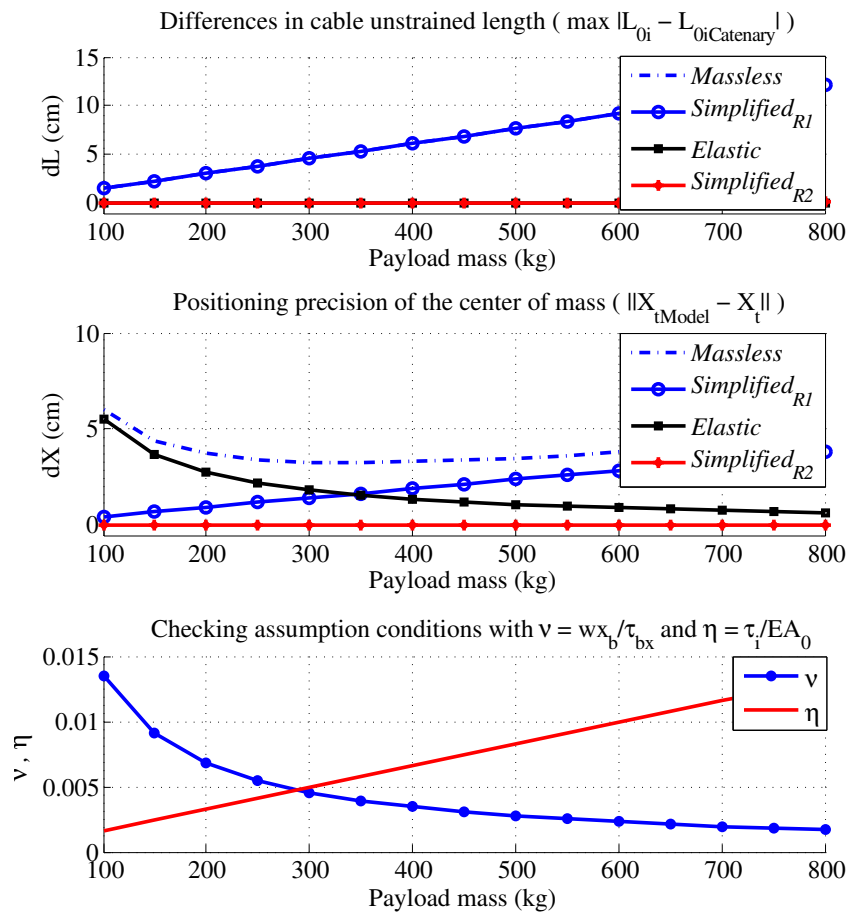

Fig. 4: Accuracies of different cable models Case $1 \mathrm{~b}$ : varying payloads

To check the assumption $\tau_{s} \ll E A_{0}$ (see Section III), we compute also the ratio

$$
\eta_{\max }=\max _{1 \leq i \leq m} \frac{\tau_{i}}{E A_{0}}
$$

The method presented in [20] is used to solve the tension distribution problem in case of massless cable model and simplified static analysis [13]. The results are then compared to the one obtained by means of the elastic catenary cable model which is considered to be the reference.

Figure 3 shows that hefty cable models lead to significant improvements compared to the massless cable model in solving the inverse kinematics (unstrained cable length). The massless cable model and simplified hefty cable model which has expression of $L_{0 i}$ with order 1 of $\mathbf{R}$ in (31) (Simplified S $_{1}$ in Fig. 3) have a similar accuracy. The simplified cable model which uses the expression of $L_{0 i}$ with order 2 of $\mathrm{R}$ in (31) (considering both cable mass and cable elasticity, Simplified $_{R 2}$ in Fig. 3) gives the best accuracy. Note that, in term of solving the inverse kinematics, elastic cable model and Simplified ${ }_{R 2}$ give similar results. It means that the elasticity has a large impact on the cable lengths. This is reasonable for the steel cables with the given characteristics (the term $E A_{0}$ is relatively small).

In the second test, the center of mass of the mobile platform of the robot is fixed at $P_{c}=\left(\begin{array}{lll}0 & 0 & 0\end{array}\right)$ in the Cartesian space. The payload of the mobile platform is varied in the range $100 \leq m_{p} \leq 800 \mathrm{~kg}$. Figure 4 shows that the cable model Simplified S2 $_{2}$ gives the best results. The impact of elasticity becomes more visible when the payload increases.

Now, let us consider the two assumptions suggested in 
Section III for the simplified cable models to be valid. The evolution of the two variables $\nu_{\max }$ and $\eta_{\max }$ in Fig. 4 shows a tradeoff of the simplified models. When the payload is small, $\eta_{\max }$ is small, thus the first assumption $\tau_{s} \ll E A_{0}$ is valid but $\nu_{\max }$ is large, hence the second assumption $\nu \ll$ 1 is violated and vice versa. According to this example, it can be seen that the validity of simplified cable model is a difficult issue when solving kinetostatic problems of CDPR.

\section{B. Experiments}

In our tests, the sagging effect of the cables is only considered between the end-points $A_{i}$ and $B_{i}$. The cable segments from end-points $A_{i}$ to the winch drums are vertical (no sagging) and assumed unstressed (the deformation effects are compensated by adding some offsets to the cable lengths). The total mass of the mobile platform in our tests is $m_{p}=210 \mathrm{~kg}$. The position of the center of mass $P_{c}$ of the mobile platform is measured by a laser tracker. The cable characteristics are given in Section VI-A. The tension distribution problem is solved by using the method in [20] based on static analysis of [13].

The robot CoGiRo is controlled by means of the scheme presented in [21]. In the latter, inverse kinematics is used to compute the desired cable unstrained lengths. To this end, (31) has been used in the experiments reported in the present paper.

The robot positioning accuracy is evaluated at several static equilibrium poses inside its workspace. Figure 5 shows the positions of these points (top view). The criteria (34), (35) and (36) are used to verify the results. In Tab. I, $X_{L}, X_{E}, X_{R 1}, X_{R 2}$ are the measured positions of the center of mass of the mobile platform in case of the massless, elastic, Simplified ${ }_{R 1}$ and Simplified Si $_{2}$ cable models, respectively.

Table I shows that the cable model Simplified $_{R 2}$ gives the best results. The elastic cable model has accuracies quite close to Simplified S $_{2}$. The massless cable model and Simplified $_{R 1}$ provide similar results with a lower accuracy.

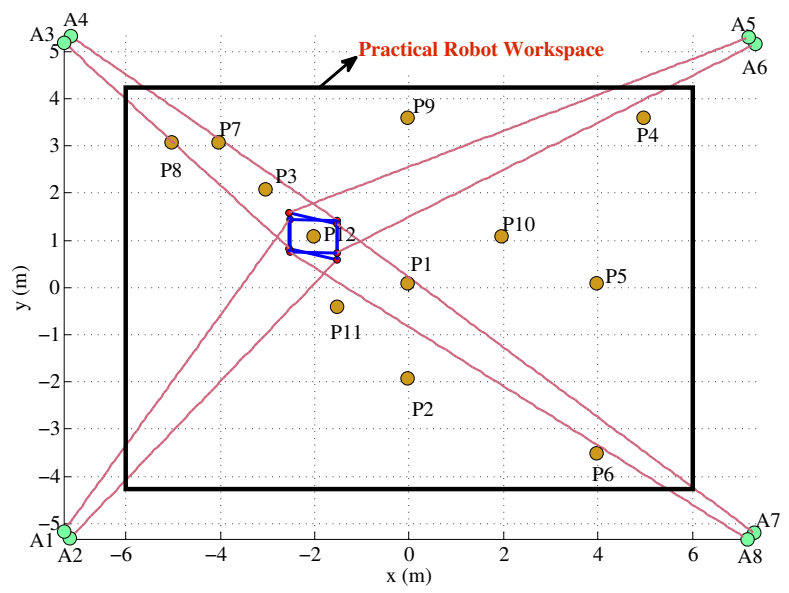

Fig. 5: Static equilibrium poses in the workspace of CoGiRo (top view)
This is reasonable since we achieved similar performances in simulation for the inverse kinematics (Fig. 3, "Differences in cable unstrained length"). In the case of the CDPR at hand (CoGiRo prototype), the differences in experimental results confirm the strong impact of the cable elasticity on the robot accuracy $\left(d X_{E}\right.$ in Tab. I), notably as compared to the cable mass alone $\left(d X_{R 1}\right)$. It remains that the best results are obtained by considering both cable mass and elasticity $\left(d X_{R 2}\right)$.

In Tab. I, at equilibrium points that are close to the center of the practical workspace ( $\nu_{\max }$ is small), we achieve good accuracies (e.g. at points $P_{1}$ and $P_{2}$ ). The accuracy of the robot reduces when the mobile platform is positioned near the workspace boundary (e.g. at points $P_{4}-P_{8}$ ). Indeed, the value of the variable $\nu_{\max }$ increases when $P_{c}$ approaches the workspace boundary (when $x_{c}$ and $y_{c}$ are large) which creates expected errors in solving the inverse kinematic problem. In addition, the variable $\eta_{\max }$ having relatively large values, it also reduces the accuracy of the simplified cable models. This fact confirms our analysis of the limitation of the simplified cable model in Section IV.

\section{CONCLUSIONS}

A simplification of the catenary cable model has been discussed in this paper. The resulting simplified cable model corresponds to the parabolic hefty cable model presented in previous studies. However, in these studies, most of the assumptions for this simplified cable model to be valid were not justified. Our approach provides a clear view of the limitation of such a simplified cable model which was verified in simulation and experiments. Indeed, the analysis of the examples in Section VI illustrated that the conditions for the simplified cable model to be valid are not always true and, hence, that they should be checked carefully.

Furthermore, a new expression of the cable unstrained length has been introduced. It accounts for both the cable mass and longitudinal elasticity. This new expression of the cable unstrained length greatly helps to improve the positioning accuracy of a large-dimension CDPR as shown by the experimental results presented in this paper. To further improve positioning accuracy, the cable characteristics, notably its Young's modulus $E$, should be experimentally identified which is part of our ongoing works.

Although this paper focus mainly on static analysis, the expression of the cable unstrained length introduced here can be useful in a dynamic analysis where the dynamics of the mobile platform and the actuators that drive the cables are taken into account. The dynamics of the cables can be neglected especially when heavy payloads are handled due to slow motions of the mobile platform. Further studies about the impacts of the presented simplified cable models on dynamic performances will be discussed in our future works.

\section{ACKNOWLEDGMENT}

The research leading to these results has received funding from the European Community's Seventh Framework Pro- 
TABLE I: Positioning accuracy of the center of mass of the mobile platform of CoGiRo at several static equilibrium poses

\begin{tabular}{|c|c|c|c|c|c|c|c|c|c|c|}
\hline $\begin{array}{c}\text { Static poses } \\
\left(x_{c}, y_{c}, z_{c}\right)(m)\end{array}$ & $\begin{array}{c}\mathrm{X}_{\mathrm{L}} \\
\left(x_{c}, y_{c}, z_{c}\right)(m)\end{array}$ & $\begin{array}{c}\mathrm{X}_{\mathrm{R} 1} \\
\left(x_{c}, y_{c}, z_{c}\right)(m)\end{array}$ & $\begin{array}{c}\mathrm{X}_{\mathrm{E}} \\
\left(x_{c}, y_{c}, z_{c}\right)(m)\end{array}$ & $\begin{array}{c}\mathrm{X}_{\mathrm{R} 2} \\
\left(x_{c}, y_{c}, z_{c}\right)(m)\end{array}$ & $\begin{array}{l}\mathrm{dX}_{\mathrm{L}} \\
(\mathrm{cm})\end{array}$ & $\begin{array}{c}\mathrm{dX}_{\mathrm{R} 1} \\
(\mathrm{~cm})\end{array}$ & $\begin{array}{l}\mathrm{dX}_{\mathrm{E}} \\
(\mathrm{cm})\end{array}$ & $\begin{array}{c}\mathrm{dX}_{\mathrm{R} 2} \\
(\mathrm{~cm})\end{array}$ & $\nu_{\max }$ & $\eta_{\max }$ \\
\hline$P_{1}(0.0,0.0,1.0)$ & $(-0.001,0.000,0.881)$ & $(-0.001,0.000,0.882)$ & $(-0.001,-0.002,0.979)$ & $(-0.000,-0.002,0.997)$ & 11.89 & 11.83 & 2.08 & 0.32 & 0.004 & 0.006 \\
\hline$P_{2}(0.0,-2.0,1.3)$ & $(0.001,-1.991,1.161)$ & $(0.001,-1.990,1.161)$ & $(-0.002,-1.993,1.275)$ & $(-0.001,-1.993,1.294)$ & 13.91 & 13.90 & 2.61 & 0.93 & 0.006 & 0.008 \\
\hline$P_{3}(-3.0,2.0,0.2)$ & $(-2.997,1.993,0.142)$ & $(-2.998,1.994,0.143)$ & $(-2.989,1.990,0.194)$ & $(-2.988,1.988,0.203)$ & 5.86 & 5.76 & 1.66 & 1.72 & 0.013 & 0.005 \\
\hline$P_{4}(5.0,3.5,0.5)$ & $(4.999,3.493,0.444)$ & $(4.999,3,489,0.448)$ & $(4.987,3.490,0.478)$ & $(4.987,3.492,0.483)$ & 5.65 & 5.28 & 2.71 & 2.33 & 0.020 & 0.005 \\
\hline$P_{5}(4.0,0.0,0.7)$ & $(3.999,0.003,0.610)$ & $(4.001,0.001,0.616)$ & $(3.988,0.003,0.672)$ & $(3.988,0.005,0.679)$ & 9.05 & 8.38 & 3.03 & 2.49 & 0.011 & 0.005 \\
\hline$P_{6}(4.0,-3.6,1.0)$ & $(4.006,-3.594,0.907)$ & $(4.007,-3.598,0.911)$ & $(3.987,-3.595,0.960)$ & $(3.987,-3.591,0.964)$ & 9.37 & 8.98 & 4.25 & 3.96 & 0.015 & 0.007 \\
\hline$P_{7}(-4.0,3.0,0.8)$ & $(-4.006,-2.990,0.732)$ & $(-4.003,-2.995,0.727)$ & $(-3.993,-2.990,0.771)$ & $(-3.993,-2.989,0.775)$ & 6.85 & 7.35 & 3.12 & 2.85 & 0.013 & 0.006 \\
\hline$P_{8}(-5.0,3.0,1.1)$ & $(-4.996,2.995,1.044)$ & $(-4.994,2.994,1.046)$ & $(-4.984,2.988,1.081)$ & $(-4.985,2.987,1.083)$ & 5.63 & 5.48 & 2.73 & 2.65 & 0.013 & 0.006 \\
\hline$P_{9}(0.0,3.5,0.9)$ & $(-0.004,3.481,0.816)$ & $(-0.003,3.481,0.824)$ & $(-0.001,3.490,0.902)$ & $(-0.002,3.490,0.904)$ & 8.61 & 7.82 & 1.01 & 1.08 & 0.018 & 0.008 \\
\hline$P_{10}(2.0,1.0,1.0)$ & $(1.999,0.995,0.896)$ & $(1.997,0.994,0.900)$ & $(1.989,0.995,0.976)$ & $(1.992,0.993,0.983)$ & 10.40 & 10.07 & 2.70 & 2.05 & 0.007 & 0.007 \\
\hline$P_{11}(-1.5,-0.5,0.8)$ & $(-1.499,-0.503,0.705)$ & $(-1.499,-0.502,-0.706)$ & $(-1.495,-0.502,0.778)$ & $(-1.495,-0.505,0.782)$ & 9.55 & 9.43 & 2.21 & 1.91 & 0.007 & 0.005 \\
\hline$P_{12}(-2.0,1.0,0.6)$ & $(-2.003,0.989,0.518)$ & $(-2.002,0.990,0.521)$ & $(-1.997,0.990,0.583)$ & $(-1.996,0.987,0.585)$ & 8.25 & 7.95 & 1.98 & 2.01 & 0.008 & 0.005 \\
\hline
\end{tabular}

gramme under grant agreement No. NMP2-SL-2011-285404 (CABLEBOT).

\section{REFERENCES}

[1] J. Albus, R. Bostelman, and N. Dagalakis, "The NIST robocrane", Journal of Robotic Systems, vol. 10, issue 5, pp. 709-724, 1993

[2] S. Havlik, "A cable-suspended robotic manipulator for large workspace operations", Comput. aided Civil Infrastruct. Eng., vol. 15, issue 6, pp. $56-68,2000$

[3] A. B. Alp and S. K. Agrawal, "Cable suspended robots: design, planning and control", Proceedings of IEEE International Conference on Robotics and Automation, ICRA 2002, pp. 4275-4280, 2002

[4] S.-R. Oh and S. K. Agrawal, "The feasible workspace analysis of a set point control for a cable-suspended robot with input constraints and disturbances", IEEE Transactions on Control Systems Technology, vol. 14, no. 4, pp. 735-742, 2006

[5] Samuel Bouchard,Clément M.Gosselin, "Kinematic sensitivity of a very large cable-driven parallel mechanism", ASME International Design Engineering Technical Conferences, 2006

[6] P. Bosscher, "Cable-suspended robotic contour crafting system", Automation in Construction, vol. 17, pp. 45-55, 2006

[7] K. Kozak, Q. Zhou, and J. Wang, "Static analysis of cable-driven manipulators with non-negligible cable mass", IEEE Transactions on Robotics, vol. 22, issue 3, pp. 325-433, 2006

[8] Mahir Hassan, Amir Khajepour, "Analysis of Large-Workspace CableActuated Manipulator For Warehousing Applications", ASME International Design Engineering Technical Conference, 2009

[9] B. Duan, Y. Qiu, F. Zhang, B. Zi, "On design and experiment of the feed cable-suspended structure for super antenna", Mechatronics, vol. 19 , pp. 503-509, 2009

[10] N. Riehl, M. Gouttefarde, S. Krut, C. Baradat and F. Pierrot, "Effects of non-negligible cable mass on the static behavior of large workspace cable-driven parallel mechanisms", IEEE International Conference on Robotics and Automation, pp. 2193-2198, 2009

[11] N. Riehl, M. Gouttefarde, C. Baradat, F. Pierrot, "On the determination of cable characteristics for large dimension cable-driven parallel mechanisms", IEEE International Conference on Robotics and Automation, ICRA 2010, pp. 4709-4714, 2010

[12] Rui Yao, Xiaoqiang Tang, Jinsong Wang, Peng Huang, "Dimensional Optimization Design of the Four-Cable-Driven Parallel Manipulator in FAST", IEEE/ASME Transaction on Mechatronics, vol. 15, issue 6, pp. 932-941, 2010

[13] M. Gouttefarde, J. Collard, N. Riehl, C. Baradat, "Simplified static analysis of large-dimension parallel cable-driven robots", IEEE International Conference on Robotics and Automation, pp. 2299-2305, 2012

[14] Hui Li, Xinyu Zhang, Rui Yao, Jinghai Sun, Gaofeng Pan and Wenbai Zhu, "Optimal Force Distribution based on slack rope model in the incompletely constrained cable-driven parallel mechanism of FAST telescope", Cable-Driven Parallel Robots, Mechanism and Machine Science, Springer vol. 12, pp. 87-102, 2013

[15] Rui Yao, Hui Li, Xinyu Zhang, "A Modeling Method of the Cable Driven Parallel Manipulator for FAST", Cable-Driven Parallel Robots, Mechanism and Machine Science, Springer vol. 12, pp. 423-436, 2013

[16] Max Irvine, "Cable Structures", Cambridge, MA: MIT Press, 1981

[17] Huu-Tai Thai, Seoung-Eock Kim, "Nonlinear static and dynamic analysis of cable structures", Finite Elements in Analysis and Design, vol. 47, issue 3, pp. 237-246, 2011

[18] M. Gouttefarde, D. Daney, J.-P. Merlet, "Interval-Analysis-Based Determination of the Wrench-Feasible Workspace of Parallel CableDriven Robots", IEEE Transactions on Robotics, Vol. 27, No. 1, pp. $1-13,2011$

[19] L. Mikelsons, T. Bruckmann, D. Schramm, and M. Hiller, "A realtime capable force calculation algorithm for redundant tendon-based parallel manipulators", IEEE International Conference on Robotics and Automation, pp. 3869-3874, 2008

[20] Johann Lamaury, Marc Gouttefarde, "A tension distribution method with improved computational efficiency", Cable-Driven Parallel Robots, Mechanisms and Machine Science, Springer vol. 12, pp. 71-85, 2013

[21] Johann Lamaury, Marc Gouttefarde, "Control of a Large Redundantly Actuated Cable-Suspended Parallel Robot", Proceedings of the IEEE International Conference on Robotics and Automation (ICRA), 2013

[22] Wisama Khalil, Étienne Dombre, "Modeling, Identification And Control of Robots", Hermes Penton, 2002 